
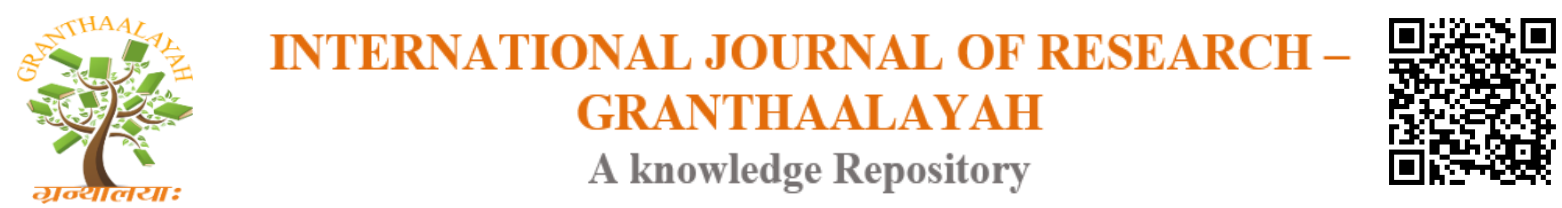

Science

\title{
THE COMMUTATIVITY OF PRIME NEAR RINGS
}

\author{
Afrah Mohammad Ibraheem *1 \\ ${ }^{* 1}$ Department of Mathematics, College of Education, Al-Mustansiriyah University, Iraq
}

\begin{abstract}
Let $\mathrm{N}$ be a near-ring, and $\sigma$ be an automorphisms of $\mathrm{N}$. An additive mapping $\mathrm{d}$ from a near-ring $\mathrm{N}$ into itself is called a reverse $\sigma$-derivation on $\mathrm{N}$ if $\mathrm{d}(\mathrm{xy})=\mathrm{d}(\mathrm{y}) \mathrm{x}+\sigma(\mathrm{y}) \mathrm{d}(\mathrm{x})$, holds for all $\mathrm{x}, \mathrm{y} \in$ $\mathrm{N}$. In this paper, we shall investigate the commutativity of $\mathrm{N}$ by a reverse $\sigma$-derivation $\mathrm{d}$ satisfied some properties, when $\mathrm{N}$ is a prime ring.
\end{abstract}

Keywords: Prime Near Ring; Reverse Derivation; Reverse $\Sigma$-Derivation; Commutativity.

Mathematics Subject Classification: 16W25, 16Y30, $16 \mathrm{U} 80$.

Cite This Article: Afrah Mohammad Ibraheem. (2018). "THE COMMUTATIVITY OF PRIME NEAR RINGS." International Journal of Research - Granthaalayah, 6(2), 339-345. https://doi.org/10.5281/zenodo.1199133.

\section{Introduction}

Near-rings are one of the generalize structures of rings. A near-ring $N$ is a ring $(N,+,$.$) , where +$ is not necessarily abelian and with only one distributive law. A left near-ring (resp. right nearring) is called a zero-symmetric left near-ring (resp. a zero-symmetric right near-ring) if $0 x=0$ (resp. $x 0=0$ ), for all $x \in N$. A near-ring $N$ called a prime near-ring if $x N y=0$ implies $x=0$ or $y=0$, for all $x, y \in N$. The multiplicative center $Z$ of $N$ will denote, $Z=\{x \in N: x y=y x$ for all $y \in N\}$. The symbol $[x, y]$ will denote the commutator $x y-y x$, for all $x, y \in N$, and note that important identities $[x, y z]=y[x, z]+[x, y] z$ and $[x y, z]=x[y, z]+[x, z] y$ satisfied for all $x, y, z \in N$. An additive mapping $d: N \rightarrow N$ is called derivation if $d(x y)=x d(y)+d(x) y$, or equivalently (cf.[7]) that $d(x y)=d(x) y+x d(y)$, for all $x, y \in N$. The derivation $\mathrm{d}$ will called commuting if $[d(x)$, $x]=0$, for all $x \in N$. The study of commutativity of prime near-rings by using derivations was initiated by H. E. Bell and G. Mason in 1987 [2], and Yilun Shang [8] satisfying the commutativity of prime near rings $N$ if there exist $k, l \in N$ such that $N$ admits a generalized derivation $D$ satisfying either $D([x, y])=x k[x, y] x l$ for all $x, y \in N$ or $D([x, y])=-x k[x, y] x l$ for all $x$, $y \in N$.In [5] A. A. M. Kamal generalizes some results of Bell and Mason by studying the commutativity of 3-prime near-rings using a $\sigma$-derivation instead of the usual derivation, where $\sigma$ is an automorphism on the near-ring. Bresar and Vukman in 1989 [3] have introduced the notion of a reverse derivation as an additive mapping $\mathrm{d}$ from a ring $R$ into itself satisfying $d(x y)=d(y) x$ $+y d(x)$, for all $x, y \in R$. Samman and Alyamani [6] studied the reverse derivations on semi prime 
rings. C.Jaya S. R., G.Venkata B.Rao and S.Vasantha Kumar in [4] studied generalized reverse derivation of a semi prime ring $R$ and proved that if $\mathrm{f}$ is a generalized reverse derivation with a derivation $d$, then $\mathrm{f}$ is a strong commutativity preserving and $R$ is commutative. Afrah M.Ibraheem in [1] used the notion of reverse derivations on a prime $\Gamma$-near $\operatorname{ring} M$ to study the commutativity conditions of $M$, when $U$ be a non-zero invariant subset of $M$. In this paper, we shall prove that a prime near-ring which admits a nonzero reverse $\sigma$-derivation satisfying certain conditions must be a commutative ring. Throughout the paper $N$ will denote a zero symmetric near-ring with multiplicative center $Z$.

\section{Preliminary Results}

To prove our results we start with the following definition and lemmas:

\section{Definition 2.1:}

Let $N$ be a near-ring, and $\sigma$ is an automorphism on $N$. An additive mapping $d$ from $N$ into itself is called a reverse $\sigma$-derivation on $N$ if satisfying $d(x y)=d(y) x+\sigma(y) d(x)$, for all $x, y \in N$.

\section{Lemma 2.2:}

Let $d$ be an arbitrary additive automorphism of $N$. Then $d(x y)=\sigma(y) d(x)+d(y) x$ for all $x, y \in N$ if and only if $d(x y)=d(y) x+\sigma(y) d(x)$, for all $x, y \in N$. Therefore $d$ is a reverse $\sigma$-derivation if and only if $d(x y)=d(y) x+\sigma(y) d(x)$.

Proof: Suppose

$d(x y)=\sigma(y) d(x)+d(y) x$,

for all $x, y \in N$. Since

$(x+x) y=x y+x y$,

$d((x+x) y)=d(x y+x y)$

$d((x+x) y))=\sigma(y) d(x+x)+d(y)(x+x)$.

$=\sigma(y) d(x)+\sigma(y) d(x)+d(y) x+d(y) x \ldots$

for all $x, y \in N$. And,

$d(x y+x y)=d(x y)+d(x y)$

$=\sigma(y) d(x)+d(y) x+\sigma(y) d(x)+d(y) x \ldots$

for all $x, y \in N$. From (1) and (2), we get

$\sigma(y) d(x)+d(y) x=d(y) x+\sigma(y) d(x)$,

So,

$d(x y)=d(y) x+\sigma(y) d(x)$,

for all $x, y \in N$. The converse is similarly. 


\section{Lemma 2.3:}

Let $N$ be a prime near-ring, and $d$ be a nonzero reverse $\sigma$-derivation of $N$. If $d(N) \subset Z(N)$ then $N$ is a commutative ring.

\section{Proof}

Let $d(x) \in Z(N)$, for all $x \in N$. Then

$d(X) z=z d(X)$...

Replacing $x$ by $x y$ in (1), we have

$(d(y) x+\sigma(y) d(x)) z=z(d(y) x+\sigma(y) d(x))$,

Then

$\sigma(y) d(x) z-z \sigma(y) d(x))=-d(y) x z+z d(y) x,$.

$=-d(y) x z+d(y) z x \ldots$

for all $x, y \in N$. Replacing $\sigma(y)$ by $d(x)$ in (2) and using (1), we get

$d(y)(-x z+z x)=d(y)[-x, z]$

$=d(y)[z, x]=0 \ldots$

for all $x, y, z \in N$. Replacing $z$ by $z y$ in (3) and using (3) again, we get

$d(y) z[y, x]=0$,

for all $x, y, z \in N$. Since $N$ is a prime, and $d \neq 0$, we have

$[y, x]=0$,

for all $x, y \in N$. Therefore $\mathrm{N}$ is commutative.

\section{Lemma 2.4:}

Let $N$ be a prime near-ring with center $Z$, and let $d$ be a nonzero reverse $\sigma$-derivation of $N$, then $d(Z) \subset Z$.

Proof: For any $z \in Z$ and $x \in N$, we have

$d(X z)=d(z X)$.

$d(x z)=d(z) x+\sigma(z) d(x)$

$=\sigma(z) d(x)+d(z) x$,

by lemma 2.2. If we replace $\sigma(z)$ by $z$, we get

$d(x z)=z d(x)+d(z) x \ldots$

for all $x, z \in N$.

$d(z x)=d(x) z+\sigma(x) d(z) \ldots$

for all $x, z \in N$. From (1) and (2), we get

$d(z) x=\sigma(x) d(z)$, 
and since $\sigma$ is automorphism, we have

$d(z) x=x d(z)$,

for all $x, z \in N$, Therefore $d(z) \in Z$, this complete proof.

\section{Lemma 2.5:}

Let $d$ be a nonzero reverse $\sigma$-derivation of a prime near-ring $N$, and $x \in N$.

If $x d(N)=0$ or $d(N) x=0$, then $x=0$.

Proof: Let assume that,

$x d(n)=0 \ldots$

for all $n \in N$. Replacing $n$ by $m n$ in (1), we have

$x d(n) m+x \sigma(n) d(m)=0 \ldots$

for all $x, n, m \in N$. By using (1) in (2), and since $\sigma$ is automorphism, we have $x N d(m)=0$,

for all $x, m \in N$, and since $N$ is a prime, and $d(N) \neq 0$, we have $x=0$.

Similarly, we can prove $x=0$, if $d(N) x=0$.

\section{The Commutativity of Prime Near Ring $N$}

In this section we give conditions under which a prime near ring $N$ must be commutative ring.

\section{Theorem 3.1:}

For a prime near ring $N$, let $d$ be a nonzero reverse $\sigma$-derivation of $N$, such that $[x, d(x)]=0$, for all $x \in N$, then $N$ is commutative.

Proof: Let

$[x, d(x)]=0 \ldots$

for all $x \in N$. Replacing $d(x)$ by $y d(x)$ in (1) and using (1) again, we have $[x, y] d(x)=0 \ldots$

for all $x, y \in N$. Replace $y$ by $z y$ in equ.(2) and using (2), we get, $[x, z]$ y $d(x)=0$,

for all $x, y, z \in N$.Since $N$ is a prime, we have either $[x, z]=0$ or $d(x)=0$.

Since $d(x) \neq 0$, for all $x \in N$, then we have $[x, z]=0$, it follows that $x \in Z(N)$ for each fixed $x \in N$, and by lemma 2.4, we get $d(x) \in Z(N)$, that's $d(N) \subset Z(N)$. Then by lemma 2.3, we get $N$ is commutative. 


\section{Theorem 3.2:}

Let $N$ be a prime near ring, and $d$ be a nonzero reverse $\sigma$-derivation of $N$. If $[d(y), d(x)]=0$, for all $x, y \in N$, then $N$ is commutative.

Proof: Given that

$[d(y), d(x)]=0 \ldots$

for all $x, y \in N$. Replacing $y$ by $y x$ in (1), we get,

$[d(x) y+\sigma(x) d(y), d(x)]=0$,

By using (1) again, we get

$d(x)[y, d(x)]+[\sigma(x), d(x)] d(y)=0 \ldots$

for all $x, y \in N$. Replacing $y$ by $z y$, where $z \in Z(N)$ in equ.(2), we get,

$d(x) z[y, d(x)]+d(x)[z, d(x)] y+[\sigma(x), d(x)] d(y) z+[\sigma(x), d(x)] \sigma(y) d(z)=0 \ldots$

for all $x, y, z \in N$. Since $\sigma$ is automorphism, and by using (2) in (3), we get $[\sigma(x), d(x)] y d(z)=0$,

for all $x, y, z \in N$. Since $N$ is a prime, we have either

$[\sigma(x), d(x)]=0$, or $d(z)=0$.

Since $\mathrm{d}(\mathrm{z}) \neq 0$, we have

$[\sigma(x), d(x)]=0 \ldots$

for all $x \in N$. Replacing $\sigma(x)$ by $x$ in (4), and by using the similar procedure as in Theorem 3.1, we get, $N$ is commutative.

\section{Theorem 3.3:}

Let $N$ be a prime near ring, and $d$ be a nonzero reverse $\sigma$-derivation of $N$. If $[x, d(y)] \in Z(N)$, for all $x, y \in N$, then $N$ is commutative.

Proof: Assume that

$[x, d(y)] \in Z(N)$,

for all $x, y \in N$. Hence for all $n \in N$,

$[[x, d(y)], n]=0 \ldots$

Replacing $x$ by $x d(y)$ in (1), and using (1) again, we get

$[x, d(y)][d(y), n]=0 \ldots$

for all $x, y, n \in N$. 
Replacing $x$ by $n x$ in (2), and using (2) again, we get

$[n, d(y)] x[d(y), n]=0 \ldots$

for all $x, y, n \in N$. Since $N$ is a prime, we have either

$[n, d(y)]=0 \ldots$

for all $y, n \in N$, or

$[d(y), n]=0 \ldots$

for all $y, n \in N$. If we replacing $d(y)$ by $m d(y)$ in (4) and (5), and using them again, we get $[n, m] d(y)=0$

or

$$
[m, n] d(y)=0
$$

for all $y, n, m \in N$. By using lemma 2.5 in two cases, we have

$$
[n, m]=0 \text {, and }[m, n]=0 \text {, }
$$

for all $n, m \in N$. Therefore, $N$ is commutative.

\section{Theorem 3.4:}

Let $N$ be a prime near ring, $d$ be a nonzero reverse $\sigma$-derivation of $N$, and $y \in N$. If $[d(x), y]=0$ then $d(y)=0$ or $y \in Z(N)$.

Proof: Let

$[x, d(x)]=0 \ldots$

for all $x \in N$. Replacing $d(x)$ by $y d(x)$ in (1) and using (1) again, we have

$[x, y] d(x)=0 \ldots$

For all $x, y \in N$. Replace $y$ by $z y$ in equ.(2) and using (2), we get,

$[x, z]$ y $d(x)=0$,

For all $x, y, z \in N$. Since $N$ is a prime, we have either $[x, z]=0$ or $d(x)=0$.

Since $d(x) \neq 0$, for all $x \in N$, then we have $[x, Z]=0$, it follows that $x \in Z(N)$ for each fixed $x \in N$, and by lemma 2.4 , we get $d(x) \in Z(N)$, that's $d(N) \subset \mathrm{Z}(\mathrm{N})$. Then by lemma 2.3, we get $N$ is commutative.

\section{Theorem 3.5:}

Let $N$ be a prime near ring, and $d$ be a nonzero reverse $\sigma$-derivation of $N$, such that $d([x, y])=[x, d(y)]$, for all $x, y \in N$, then $N$ is commutative. 
Proof: Given that

$d([x, y])=[x, d(y)] \ldots$

for all $x, y \in N$. Replacing $y$ by $y x$ in (1) and using (1), we get,

$[x, d(x)] y+[x, \sigma(x)] d(y)=0 \ldots$

for all $x, y \in N$. If we replacing $\sigma(x)$ by $x$ in (2), we have

$[x, d(x)] y=0 \ldots$

for all $x, y \in N$. Replacing $y$ by $y d(x)$ in (3), we get

$[x, d(x)]$ y $d(x)=0$,

for all $x, y \in N$. Since $N$ is a prime, and $d \neq 0$, we have

$[x, d(x)]=0$,

for all $x \in N$. Then by theorem 3.1, we get, $N$ is commutative.

\section{Conclusions}

For an automorphism $\sigma$ on a near ring $N$, we study the commutativity on $N$, if $N$ has a non zero reverse $\sigma$-derivation $d$, where $d$ is defined as an additive mapping from $N$ into itself satisfying $d(x y)=d(y) x+\sigma(y) d(x)$, for all $x, y \in N$, and introduced some conditions on $d$ to get the commutativity on $N$ when $N$ is a prime near ring.

\section{Acknowledgements}

The author would like to thank the referees for useful comments and suggestions.

\section{References}

[1] M. Afrah Ibraheem, (2015). "Reverse Derivations on Prime Gamma Near Rings", Int. J. Pure Appl. Sci. Technol., 26(2), 64-69.

[2] H. E. Bell and G. Mason, (1987). "On derivations in near-rings, Near-rings and near-fields (Tubingen, 1985)”, North-Holland Math. Stud., 137, North-Holland, Amsterdam, 31-35.

[3] M. Bresar and J. Vukman, (1989). "On some additive mappings in rings with involution", A equations math., 38, 178-185.

[4] C.Jaya Subba Reddy, G. Venkata Bhaskara Rao, S.Vasantha Kumar, (2015). "Generalized Reverse Derivations on Semi prime Rings", The Bulletin of Society for Mathematical Services, Vol. 15, 1-4.

[5] A. A. M. Kamal, (2001). " $\sigma$-derivations on prime near-rings", Tamkang J. Math. 32, 89-93.

[6] M. Samman, N. Alyamani, (2007). "Derivations and reverse derivations in semi prime rings", International Mathematical, Forum, 2, No. 39, 1895-1902.

[7] X. K.Wang, (1994). "Derivations in prime near-rings", Proc. Amer. Math. Soc., 121(2), 361-366.

[8] Yilun Shang, (2015). "A Note on the Commutativity of Prime Near-rings", Algebra Colloquium, 22(3), 361-366.

*Corresponding author.

E-mail address: afrah.wisam@ uomustansiriyah.edu.iq 